\title{
CT Perfusion: More Than What You Thought
}

A cute stroke treatment has dramatically changed in the past decade. Boundaries are crossed, and previous axioms are no longer relevant. The results of the DWI or CTP Assessment with Clinical Mismatch in the Triage of Wake-Up and Late Presenting Strokes Undergoing Neurointervention with Trevo (DAWN) and Endovascular Therapy Following Imaging Evaluation for Ischemic Stroke 3 (DEFUSE-3) trials, ${ }^{1,2}$ examining endovascular thrombectomy (EVT) in the late timeframe (6-24 hours from stroke onset), have thrust perfusion imaging into the evaluation of acute stroke protocol. ${ }^{3}$ The primary purpose of CTP is to distinguish salvageable tissue from infarct core and thus improve patient selection for EVT. $^{3-5}$ It is now widely used as part of an acute stroke CT protocol. However, many stroke centers worldwide routinely perform CTP only for patients beyond the early timeframe. This is in accordance with the recent acute stroke guidelines, recommending CTP in the late (6-24 hour) timeframe. ${ }^{6}$

CTP offers other diagnostic utilities. ${ }^{7}$ First, CTP may help differentiate ischemic stroke from stroke mimics, including seizure, migraine aura, and conversion disorder. ${ }^{7-9}$ Although the suspicion for a stroke mimic should not be based solely on CTP, it may add supportive data and help clinicians better diagnose these elusive cases. Second, CTP may contribute valuable information for the selection of candidates for EVT and intravenous thrombolysis (IVT), despite a low NIHSS score. ${ }^{3}$ The NIHSS gives much weight to motor function and dominant hemisphere. However, various infarcts may result in neurologic sequelae, not fully revealed by the NIHSS. ${ }^{10}$ These patients may greatly benefit from IVT in case a penumbral lesion is identified. Third, CTP can demonstrate the severity of hypoperfusion in the presence of carotid occlusion or dissection and thus help determine the need for reperfusion therapy. ${ }^{7,11}$

In this issue of the American Journal of Neuroradiology, Amukotuwa et $\mathrm{al}^{12}$ examined the use of CTP to aid in interpretation of CTA for distal vessel occlusion (DVO) identification. They demonstrated that the use of time-to-maximum (Tmax) maps significantly improved the diagnostic performance for detecting DVO.

Detection of DVO has great importance. The field of endovascular thrombectomy is rapidly emerging, and evidence is growing regarding the beneficial effect of EVT on distal M2 occlusions. ${ }^{13}$ Furthermore, EVT targeting very distal occlusions including M3/
M4, A3/A4, and P3 has become feasible, and although it still lacks evidence is probably the next frontier of EVT. ${ }^{12,13}$ Such DVOs are likely to be considered for EVT, especially in patients with disabling neurological deficit ineligible for IVT. This emphasizes the importance of fast and accurate DVO detection.

The identification of DVOs may be challenging due to their small caliber, large number, and lower resolution compared with large vessels. ${ }^{12}$ Systematic manual interrogation of these vessels is complex and time-consuming. The authors show that the use of Tmax increased the rate of DVO detection, the level of confidence, and the speed of interpretation. These beneficial effects were greater for more distal occlusions, though improved detection of M2 occlusions was also demonstrated, emphasizing the clinical relevance. The results were consistent across both experienced and inexperienced raters. The added benefit of CTP use may be especially valuable for inexperienced radiologists in remote hospitals or during night shifts. Furthermore, CTP may give the sole hint of a DVO with the increased use of automated interpretation software, which has yet to identify a DVO on CTA.

The limitations of the study arise mainly from the use of a single CTP feature and a single postprocessing software package. The authors discuss these limitations, including the possibility of false-positive results due to nonterritorial Tmax delay. Future studies are needed to compare various types of CTP software and different Tmax cutoff values and to examine the use of Tmax versus other parameters such as CBF, CBV, and MTT.

Currently, DWI is the most accurate technique for infarct detection. ${ }^{14}$ Nevertheless, CT has some main advantages over MR imaging, including the wider availability, rapid imaging, superior vascular imaging of extracranial and distal intracranial vessels, and fewer absolute contraindications. ${ }^{14}$

CTP has several limitations. ${ }^{4,5,15}$ There is still no standardization of CTP acquisition protocol or postprocessing techniques and thresholds. ${ }^{5}$ These thresholds are usually defined on the basis of the normal values of $\mathrm{CBF}$ and $\mathrm{CBV}$ of the gray matter and may be inaccurate in examining white matter lesions. ${ }^{4,15}$ Furthermore, in most cases, CTP has limited coverage and low sensitivity for the detection of lacunar infarcts. ${ }^{4,7,15}$ It may also be susceptible to the influence of impaired cardiac output and carotid artery stenosis. ${ }^{4,7}$ Therefore, obtaining accurate interpretation can be challenging, and CTP 
should be interpreted with caution, acknowledging both its advantages and pitfalls.

In the near future, the use of whole-brain CTP acquisitions on modern multidetector array CT scanners and high-resolution and standardized algorithms will optimize the diagnostic accuracy of CTP, allowing the detection of small lacunar infarcts and lowering the probability of false-negative results. ${ }^{7}$

Current guidelines recommend the use of CTP for patient selection for EVT in the late timeframe (6-24 hours). ${ }^{6}$ However, as CTP gains more diagnostic utilities, we should broaden the use of CTP and include it as a routine part of the CT stroke protocol. The results of the current study demonstrate one important advantage of CTP for patients in the early timeframe: The use of perfusion imaging adds valuable information and improves interpretation, resulting in better treatment of patients with stroke.

\section{REFERENCES}

1. Nogueira RG, Jadhav AP, Haussen DC, et al; DAWN Trial Investigators. Thrombectomy $\mathbf{6}$ to 24 hours after stroke with a mismatch between deficit and infarct. N Engl J Med 2018;378:1121 CrossRef Medline

2. Albers GW, Marks MP, Kemp S, et al; DEFUSE 3 Investigators. Thrombectomy for stroke at $\mathbf{6}$ to 16 hours with selection by perfusion imaging. N Engl J Med 2018;378:708-18 CrossRef Medline

3. Campbell BC, Majoie CB, Albers GW, et al; HERMES Collaborators. Penumbral imaging and functional outcome in patients with anterior circulation ischaemic stroke treated with endovascular thrombectomy versus medical therapy: a meta-analysis of individual patient-level data. Lancet Neurol 2019;18:46-55 CrossRef Medline

4. Vagal A, Wintermark M, Nael K, et al. Automated CT perfusion imaging for acute ischemic stroke: pearls and pitfalls for real-world use. Neurology 2019;93:888-98 CrossRef Medline

5. Heit JJ, Wintermark M. Perfusion computed tomography for the evaluation of acute ischemic stroke: strengths and pitfalls. Stroke 2016;47:1153-58 CrossRef Medline

6. Powers WJ, Rabinstein AA, Ackerson T, et al; American Heart Association Stroke Council. American Heart Association Stroke
Council: 2018 Guidelines for the Early Management of Patients with Acute Ischemic Stroke-A Guideline for Healthcare Professionals From the American Heart Association/American Stroke Association. Stroke 2018;49:e46-110 CrossRef Medline

7. Mangla R, Ekhom S, Jahromi BS, et al. CT perfusion in acute stroke: know the mimics, potential pitfalls, artifacts, and technical errors. Emerg Radiol 2014;21:49-65 CrossRef Medline

8. Gugger JJ, Llinas RH, Kaplan PW. The role of CT perfusion in the evaluation of seizures, the post-ictal state, and status epilepticus. Epilepsy Res 2020;159:106256 CrossRef Medline

9. Granato A, D'Acunto L, Ajčević M, et al. A novel computed tomography perfusion-based quantitative tool for evaluation of perfusional abnormalities in migrainous aura stroke mimic. Neurol Sci 2020;41:3321-28 CrossRef Medline

10. Wendt M, Tütüncü S, Fiebach JB, et al. Preclusion of ischemic stroke patients from intravenous tissue plasminogen activator treatment for mild symptoms should not be based on low National Institutes of Health Stroke Scale scores. J Stroke Cerebrovasc Dis 2013;22:55053 CrossRef Medline

11. Brown SC, Falcone GJ, Hebert RM, et al. Stenting for acute carotid artery dissection. Stroke 2020;51:e3-e6 CrossRef Medline

12. Amukotuwa $\mathrm{SA}, \mathrm{Wu} \mathrm{A}$, Zhou $\mathrm{K}$, et al. Tmax improves diagnostic performance for detecting distal vessel occlusions on CT angiography. AJNR Am J Neuroradiol 2020;42:65-72 CrossRef

13. Saver JL, Chapot R, Agid R, et al; Distal Thrombectomy Summit Group. Thrombectomy for distal, medium vessel occlusions: a consensus statement on present knowledge and promising directions. Stroke 2020;51:2872-84 CrossRef Medline

14. Biesbroek JM, Niesten JM, Dankbaar JW, et al. Diagnostic accuracy of CT perfusion imaging for detecting acute ischemic stroke: a systematic review and meta-analysis. Cerebrovasc Dis 2013;35:493-501 CrossRef Medline

15. Sotoudeh H, Bag AK, Brooks MD. "Code-Stroke” CT perfusion: challenges and pitfalls. Acad Radiol 2019;26:1565-79 CrossRef Medline

(D). Molad

Department of Stroke and Neurology Tel-Aviv Sourasky Medical Center

Tel-Aviv, Israel 